\title{
Operation Risk Assessment of Power System With High Risk Wind Power Ramping
}

\author{
Tong Xinyuan, Wang Hongtao \\ Department of Electrical Engineering, Shan Dong University, Ji Nan, China \\ 1055495162@qq.com
}

\begin{abstract}
Keywords: positive and negative wind power ramp event; risk assessment; Monte Carlo simulation;
\end{abstract} wind power ramp model; power flow.

\begin{abstract}
In order to evaluate the risk of wind power curtailment and loss of load brought by wind power ramp event on power system, risk assessment model of wind power ramp event based on Monte Carlo method is established. According to the result of wind power prediction system, the wind power model of day-ahead positive and negative wind power ramp event is established. Several constraints need to be satisfied during the evaluation process. Risk indicators like expectation of wind power curtailment, loss of load expectation, times of loss load expectation and off-limit risk indices of line power flow are calculated during the process of risk assessment. A specific algorithm flow of risk assessment is also given in this article. Finally, the validation of the proposed method is verified in IEEE-RTS79. The simulation results demonstrate variation trend of risk indicators under different amplitude, start time and duration of wind power ramp events, and indicate the weak spot of power system under the circumstance of wind power ramp event.
\end{abstract}

\section{Introduction}

With the increasing penetration of wind power, the impact that the volatility of wind power bring to power grid has draw people's attention increasingly, wind power fluctuations caused by extreme weather are particularly noteworthy for operators. The study found that when tropical cyclone, tornado, strong convective weather and other inclement weather occurs, there will be a large scale wind power ramp event that lasts for few hours in the wind farm[1,2]. In the case of large scale and high centralized integrate of wind farm, wind power ramp event will bring huge impact and operational risk to the power grid, it will cause frequency drops and active power imbalance and even lead to the risk of wind power curtailment or loss of load[3,4]. So the operation risk evaluation of power system considering wind power ramp event is of great importance.

Accurate prediction of wind power ramp is the effective Prerequisite to evaluate the risk of power system brought by wind power ramp event. Current method of forecasting can mainly be divided into two types [5,6,7,8]: (1)forecasting from the perspective of meteorology (2)Classification and identification of wind power ramp events based on various types of historical data, and then modify the predicted wind power. However, affected by many factors, the current forecast of wind power ramp event remains uncertainty [9], also the level and accuracy of prediction has to be further improved, thereby affecting the accuracy of the results of the risk assessment.

Currently the risk evaluation of power system with wind power is mostly concentrated in assessing the reliability of power system with wind power in a relatively large time scale $[10,11,12]$. These studies tend to focus on statistical results in a large time scale, and therefore it is difficult to adapt to the system risk evaluation considering wind power ramp event. Though existing research on assessing the risk of power system considering wind power ramp event $[13,14]$ has considered the risk of loss of load, however, there are still some minor flaws on the wind power ramp model; in addition, they failed to focus on the risk of wind power curtailment caused by positive wind power ramp event; impact of wind power ramp event on power flow is also worth attention of operators.

According to the prediction of wind power ramp event, a risk assessment model considering positive and negative wind power ramp event based on Monte Carlo method is established in this 
paper. Thus operators are able to take steps to prevent and control the ramp event. Models, constraints and risk indices are introduced. Case studies are given in the final period.

\section{Modeling and analysis of wind power ramp event}

\subsection{The uncertainty of wind power ramp event}

The uncertainty of ramp event is reflected in 3 aspects: start time, duration and amplitude. At the present stage, forecasting is the main measure to deal with the uncertainty of wind power ramp event. However, difficulty of forecasting wind power ramp event is relatively large due to the uncertainty of ramp event, and thus increased the uncertainty of prediction.

Risk evaluation of wind power ramp event is based on its prediction, the larger the prediction error is, the lower the prediction accuracy will be, therefore lead to low credibility of risk assessment results, and thus affect the decision making of operators.

2.2 Model of the wind power ramp event

The start time, duration and amplitude of wind power ramp are under great uncertainty subject to the uncertainty and low precision level of prediction with wind power ramp. The wind power output is consistent with the day-ahead prediction before and after the ramp event, thus prediction error is considered.

Wind power ramp up and ramp down model diagrams are shown in Figure 1 and Figure 2. In this model, the initial wind power is $P_{W}\left(T_{S}\right)$, wind power ramp event starts at time $T_{S}$. The duration of wind power ramp can be divided into $n$ prediction intervals, there will be a ramp amplitude $P_{\text {amp }}$ in each interval. Assume the ramp rate of wind power is unchanged in each interval, then the total duration $T_{d u r}$ and ramp amplitude $P_{\text {amp }}$ can be expressed by the time intervals and ramp amplitude of $n$ prediction intervals. The prediction error $P_{W, e r r}(t)$ is also considered before and after the wind power ramp. The model of wind power ramp event can be expressed as

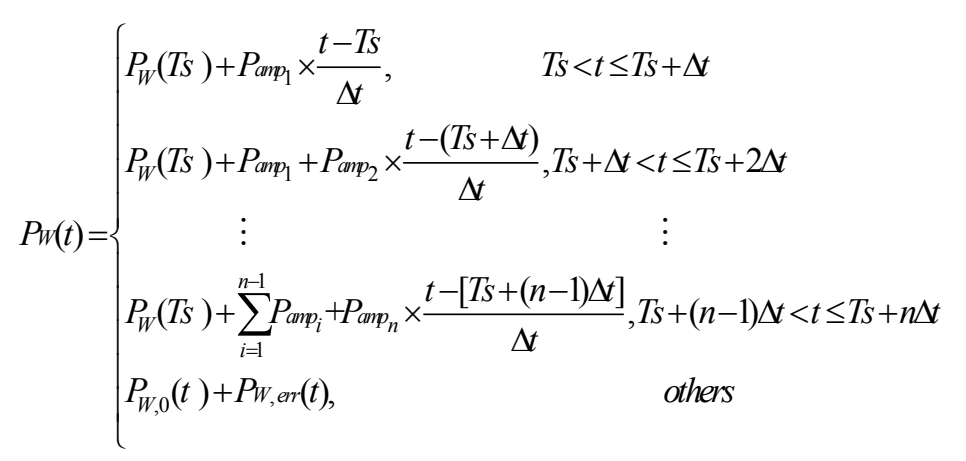

Where $P_{W}(t)$ is wind power in period $t, T_{S}$ is the start time, $P_{W}(T s)$ is the initial wind power, $P_{\text {amp }}$ is the ramp amplitude in the $i$ th prediction interval, $\Delta t$ is a prediction time interval, $P_{W, 0}(t)$ is the day-ahead prediction of wind power in period $t, P_{W, e r r}(t)$ is the prediction error in period $t$. The expectation and probabilistic distribution of start time $T_{S}$ and amplitude $P_{a m p}$ are provided by wind power ramp event forecasting system. The expectation and probabilistic distribution of $T_{S}, T_{d u r}$ and $P_{a m p}$ are obtained by means of modeling wind power ramp event.

Because 3 characteristic indicators $T_{S}, T_{d u r}$ and $P_{\text {amp }}$ are independent from each other, it is crucial to sample 3 characteristic indicators separately according to their expectation and probabilistic distribution of in the process of risk assessment. 


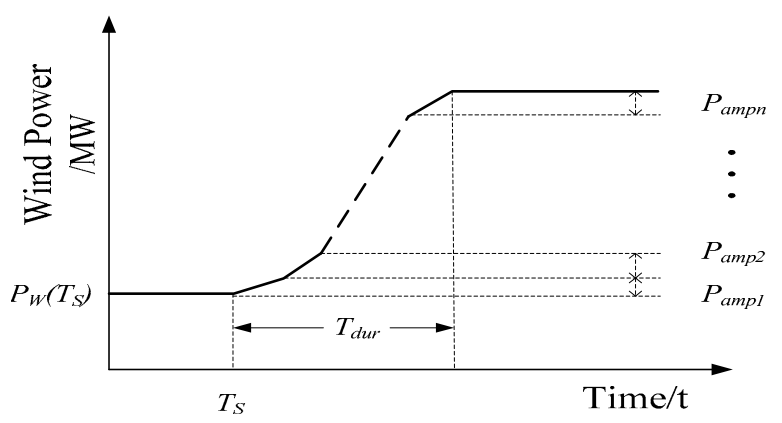

Fig. 1 Model of wind power ramp-ups

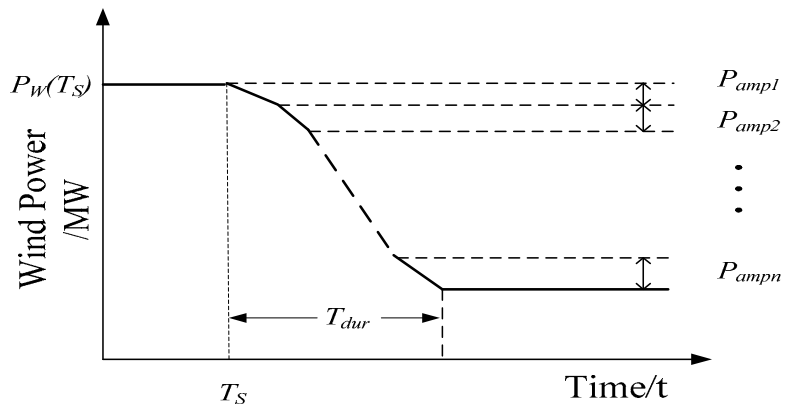

Fig. 2 Model of wind power ramp-downs

\section{Risk Evaluation of Wind Power Ramp Event}

3.1 Constraints satisfied in the system

Constraints that should be satisfied in the system during the process of risk evaluation are listed as follows:

(1) Active power balance constraint

$$
\sum_{i=1}^{N_{G}} P_{G i}^{t}+P_{W}^{t}=P_{L}^{t}
$$

(2) Conventional unit output constraint

(3) Wind power output constraint

$$
U_{i}^{t} P_{G i}^{\min } \leq P_{G i}^{t} \leq U_{i}^{t} P_{G i}^{\max }
$$

$$
0 \leq P_{W}^{t} \leq P_{W \max }^{t}
$$

(4) Conventional unit ramp rate constraint

$$
\left\{\begin{array}{l}
P_{G i}^{t-1}-D R_{G i} \leq P_{G i}^{t} \leq P_{G i}^{t-1}+U R_{G i} \\
P_{G i}^{t-1}-R_{d i}^{t-1} \leq P_{G i}^{t} \leq P_{G i}^{t-1}+R_{u i}^{t-1}
\end{array}\right\}
$$

(5) Transmission line active power constraint

$$
\left|T_{k}(S)\right| \leq T_{k}^{\max }
$$

Where $P_{G i}^{t}$ is the output of $i$ th unit in period $t ; P_{W}^{t}$ is the output of wind farm in period $t ; P_{L}^{t}$ the total load of system in period $t ; U_{i}^{t}$ means the state of the $i$ th unit in period $t$ ( 0 means off while 1 means on); $P_{G i}^{\max }$ and $P_{G i}^{\min }$ represent the maximum and minimum output of $i$ th unit respectively; $P_{W \max }^{t}$ is the maximum output of wind farm; $U R_{G i}$ and $D R_{G i}$ denote the up and down ramp rate of $i$ th unit; $R_{u i}^{t}$ and $R_{d i}^{t}$ represent the up and down reserve capacity respectively of $i$ th unit in period $t$; $T_{k}(S)$ is the active power flow vector of branch $k$ during wind power ramp, $T_{k}^{\max }$ is the limit of $T_{k}(S)$. 3.2 Risk indicators 
When extreme weather events occur, if the wind speed does not exceed the cut off limit of wind turbine, the wind power will often rise by a large margin in the first place, the net load will fall by a large margin in a short period of time. If the negative ramp rate and down reserve capacity of conventional units are insufficient so that it cannot track the sharp fall of the net load, the phenomenon of wind energy curtailment will appear and create corresponding punishment on the economy. When the wind speed exceeds the cut off limit caused by extreme weather, a ramp down event will occur, the net load will rise by a large margin in a short period of time. If the positive ramp rate and up reserve capacity of conventional units are insufficient so that it cannot track the sharp rise of the net load, the phenomenon of loss of load will appear.

(1) Risk indicators of wind energy curtailment

In order to evaluate the risk of wind power curtailment of the system caused by wind power ramp up event, EWEC (Expected Wind Energy Curtailment) is used to calculate the curtailed wind power.

$$
E W E C=\frac{1}{K} \sum_{k=1}^{K} \sum_{i=1}^{S} \Delta w c_{i, k} \Delta t_{i, k}=\frac{1}{K} \sum_{k=1}^{K} \sum_{t=1}^{T} \Delta w c k(t) \Delta t k
$$

Where $K$ is the number of sampling using Monte Carlo method, $i$ is the state that causes the event of wind energy curtailment, $S$ are the total state that cause the event of wind energy curtailment, $\Delta w c_{i, k}$ is the curtailed wind power at $i$ th state in the $k$ th sampling, $\Delta t_{i, k}$ is the duration of state $i$ in the $k$ th sampling, $T$ is an assessment cycle.

(2) Risk indicators of loss of load

EENS (Expected Energy Not Supplied) and ENENS (Expected Number of Energy Not Supplied) are adopted to evaluate the risk of loss of load of the system caused by wind power ramp down event.

$$
\begin{gathered}
E E N S=\frac{1}{K} \sum_{k=1}^{K} \sum_{j=1}^{S} \Delta p_{j, k} \Delta t_{j, k}=\frac{1}{K} \sum_{k=1}^{K} \sum_{t=1}^{T} \Delta p_{k}(t) \Delta t_{k} \\
\text { ENENS }=\frac{1}{K} \sum_{k=1}^{K} \sum_{t=1}^{T} I_{E N S, k}(t)
\end{gathered}
$$

Where $j$ is the state that causes the event of loss of load, $\Delta p_{j, k}$ is the power shortage at $j$ th state in the $k$ th sampling, $\Delta t_{j, k}$ is the duration of state $j$ in the $k$ th sampling, $I_{E N S, k}(t)$ is the sign of load losing in the $k$ th sampling in period $t, I_{E N S, k}(t)$ equals 1 when load losing happens otherwise it equals 0 .

(3) Off-limit risk indicators of transmission line power flow

Off-limit risk indices of transmission line power flow can provide off-limit extent of transmission line power flow in the system to the operators, it can reflect the risk that wind power ramp bring to the system more thoroughly. Off-limit risk indicator of line $l$ is calculated by

$$
R_{\text {overload }, l}=\frac{1}{K} \sum_{k=1}^{K} \sum_{t=1}^{T}\left(\frac{P l(t)}{P_{l \max }}\right)^{2}
$$

Where $R_{\text {overload }, l}$ is the off-limit indicator of power flow of line $l, P_{l}(t)$ is the active power of line $l$ in period $t, P l$ max represents the limit of active power of line $l$.

\section{The Calculation Process of Wind Power Ramp Event Risk Evaluation Indexes}

In this paper, Monte Carlo method is adopted to evaluate the risk of power system considering wind power ramp event. The calculation process of the risk assessment considering wind power ramp event is shown in Figure 3. 


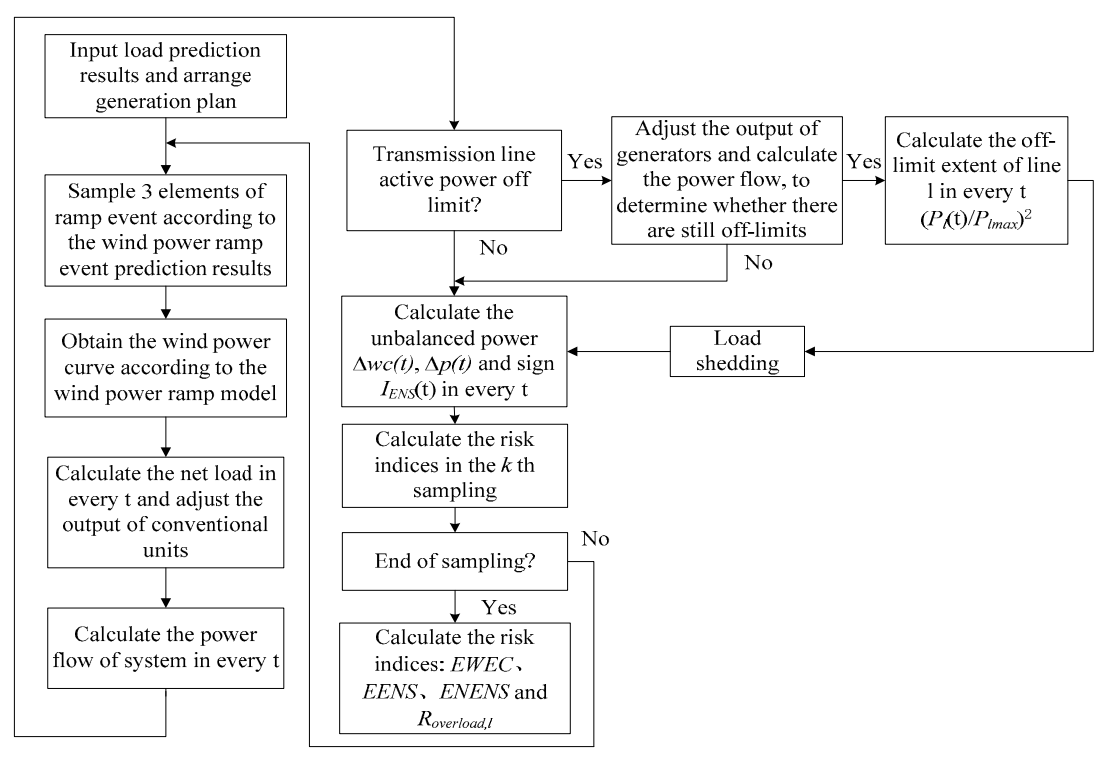

Fig. 3 Flow diagram of risk assessment model

\section{Case Studies}

Based on the above risk assessment model of wind power ramp event, the proposed method is verified in the modified IEEE-RTS79 system. Monte Carlo simulation method is adopted and corresponding codes are written in Matlab7. The system consists of 24 nodes, 32 generators and 34 transmission lines, the total capacity of the system reaches $3405 \mathrm{MW}$, and the peak load is 2850 MW. The generator with the capacity of $400 \mathrm{MW}$ in bus 18 is replaced by the wind farm of equivalent capacity, so the wind penetration is about $11.75 \%$. The day-ahead prediction result of wind power is from a wind farm of China's Shandong province. Assume the start time, duration and amplitude of the wind power ramp event follows the normal distribution. DC power flow is adopted when calculating the power flow of the system.

5.1 The variation tendency of risk indices under different scenarios

Four scenarios are demonstrated in order to study system risk caused by wind power ramp event under different scenarios:
(1) $P_{\text {amp }}=50 \%, T_{\text {dur }}=120 \mathrm{~min}$
(2) $P_{\text {amp }}=50 \%, T_{\text {dur }}=60 \mathrm{~min}$
(3) $P_{\text {amp }}=100 \%, T_{d u r}=120 \mathrm{~min}$
(4) $P_{\text {amp }}=100 \%, T_{\text {dur }}=60 \mathrm{~min}$

The impact of different wind power ramp scenarios on the system risk indices under different start time of wind power ramp are shown in Figure 4, Figure 5 and Figure 6.

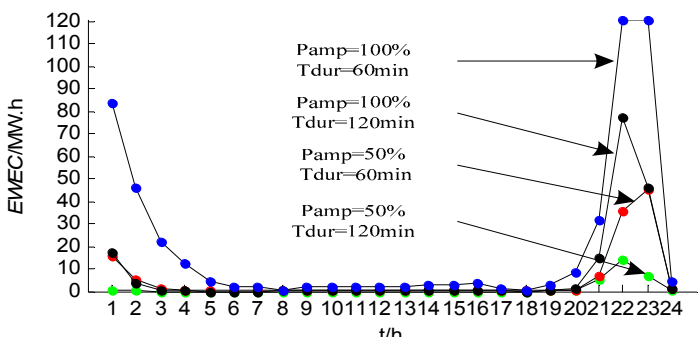

$\mathrm{t} / \mathrm{h}$

Fig.4 EWEC in different type of wind power ramp-ups 


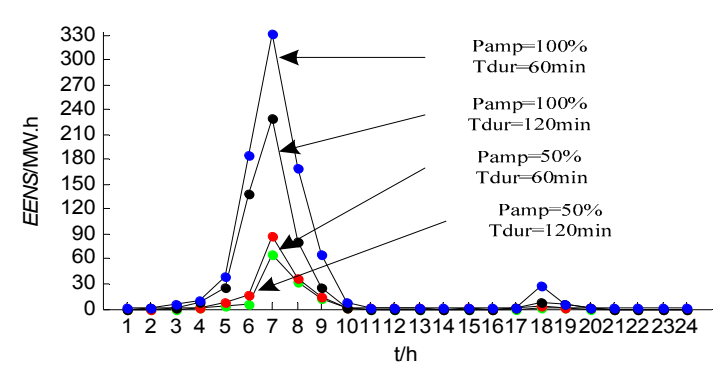

Fig.5 EENS in different type of wind power ramp-downs

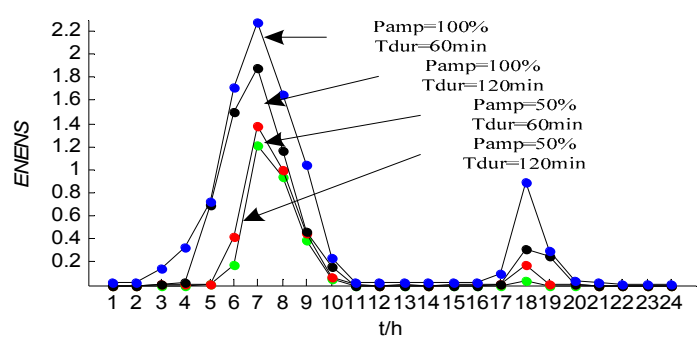

Fig.6 ENENS in different type of wind power ramp-downs

It can be seen that, the risk indices showed an increasing trend with the increasing amplitude and decreasing duration of the wind power ramp event. Moreover, the variation tendency of risk indices is basically the same as the variation tendency of the load. When the load level is relatively low, such as $1-4 \mathrm{~h}$ and $21-24 \mathrm{~h}$, the value of EWEC is relatively high accordingly, in this period the adjustable down reserve capacity of conventional units is relatively small. However when the load level is relatively high during the day time, risk indices EENS and ENENS are relatively high accordingly, in this period the adjustable up reserve capacity of conventional units is relatively small.

It is also indicated from Figure 4, Figure 5 and Figure 6 that, the difference of the start time of ramp event leads to the difference of risk indices. Changes of the system risk indices are not only related with the load level, but also closely linked with variation tendency of the net load. In Figure 4 , when the start time of ramp up event is in the period $1-4 \mathrm{~h}$ or $21-24 \mathrm{~h}$, because the load is in a downward tendency in these two periods, ramping up of the wind power leads to the increase of the drop rate of the net load, the negative ramp rate and down reserve capacity of conventional units are insufficient so that it cannot track the sharp fall of the net load, the value of EWEC is higher accordingly. While when the start time of the ramp up event is in other periods of the day, the variation tendency of the load is basically the same as the variation tendency of the wind power, and the phenomenon of dramatic decline of net load will not appear, the regulation ability of conventional units is sufficient to avoid the phenomenon of wind power curtailment. In Figure 5 and Figure 6, the event of load rise and wind power drop occur simultaneously which leads to the sharp rise of the net load during the period 5-10h and 17-19h; the positive ramp rate and up reserve capacity of conventional units are insufficient so that it cannot track the sharp rise of the net load, which leads to the increase of EENS and ENENS. While the net load shows a decline tendency in other periods of time, the phenomenon of the net load sharp rising will not appear when the start time of ramp down event is in these periods of time, thus the regulation ability of the conventional units is sufficient to avoid the phenomenon of loss of load.

5.2 Off-limit risk of transmission line power flow with wind power ramp

Risk indices of 2 transmission lines where the extent of power flow off-limit is relatively large in 6 periods of time $(5 \mathrm{~h}, 6 \mathrm{~h}, 7 \mathrm{~h}, 8 \mathrm{~h}, 9 \mathrm{~h}$, and $10 \mathrm{~h})$ the day ramp event occurs are listed in Table 1. Assume the expectation of ramp amplitude is $100 \%$, the expectation of duration is $60 \mathrm{~min}$, the expectation of start time is $6 \mathrm{~h}$. 
Table 1 Off-limit risk indices of transmission line power flow

\begin{tabular}{ccccccc}
\hline \multirow{2}{*}{$\begin{array}{c}\text { Branch } \\
\text { number }\end{array}$} & $5 \mathrm{~h}$ & $6 \mathrm{~h}$ & $7 \mathrm{~h}$ & $8 \mathrm{~h}$ & $9 \mathrm{~h}$ & $10 \mathrm{~h}$ \\
\cline { 2 - 7 } & 0 & 10.5422 & 69.7749 & 85.6256 & 88.7976 & 88.8820 \\
11 & 0 & 0 & 61.0728 & 61.1258 & 0 & 0 \\
\hline \multicolumn{2}{c}{ Note: Branch 11 and 20 are corresponding to branch7-8 and 12-13 respectively }
\end{tabular}

When the wind power ramp event occurs, the power flow off-limit risk of branch 11 and 20 is much higher than that of other branches; branch 11 and 20 are the vulnerable spot of the system, and will easily deteriorate the operation conditions of the system further when the wind power ramp event occurs. Therefore in the actual operation of the power grid, operators are supposed to focus on the real time operation of these branches; in addition, start up control measures when the off-limit risk indices of these branches exceed a certain threshold, and to ensure that the operation risk of the system is in an acceptable range.

\section{Conclusions}

A risk assessment method considering high risk wind power ramp event based on Monte Carlo simulation is proposed in this paper. Firstly, wind power model of positive and negative wind power ramp event is established considering the uncertainty of wind power ramp event. Then several constraints are considered and DC power flow is adopted to calculate the active power of transmission line. The risk indices adopted include expected wind energy curtailment, expected energy not supplied, expected number of energy not supplied and off-limit risk indices of transmission line power flow, a specific algorithm flow of risk assessment is also given in this article. Finally, the validation of the proposed method is verified in IEEE-RTS79. The simulation results demonstrate that the change of start time of wind power ramp event leads to the variation of risk indices, and the variation tendency is closely linked with the load level. The larger the ramp amplitude and the smaller the duration of wind power ramp up and ramp down events are, the greater the risk indices will be accordingly. This is because when the wind power ramp event occurs, the ramp rate and the reserve capacity of conventional units are insufficient so that the regulation ability of the conventional units are not able to track the change of the net load, thus lead to the increase of risk indices. Meanwhile the off-limit risk of transmission line power flow caused by wind power ramp event is also worth attention of operators. The evaluation results in this paper validate the effectiveness of the proposed method, thus can provide guidance for scheduling and decision making of operators.

\section{References}

[1] C. Ferreira, J. Gama, L. Matias, et al. A Survey on Wind Power Ramp Forecasting[R]. Chicago: Argonne US Department of Energy Laboratory, 2010.

[2] Kamath C. Understanding wind ramp events through analysis of historical data[C]//Transmission and Distribution Conference and Exposition, 2010 IEEE PES. IEEE, 2010: $1-6$.

[3] E. Ela, B. Kirby. ERCOT event on February 26, 2008: lessons learned[R]. Golden, Colorado: NREL, 2008.

[4] Luo $\mathrm{Xu}, \mathrm{Ma} \mathrm{Ke}$. Experience and Enlightenment in Operations of Wind Generation in ERCOT Grid[J]. Power System Technology, 2011, 35(10): 140-146.

[5] H. Zareipour, Huang Dongliang, W. Rosehart. Wind power ramp events classification and forecasting: A data mining approach[C]. IEEE Power and Energy Society General Meeting, San Diego, CA, 2011. 
[6] J. W. Zack, S. Young, M. Cote, et al. Development and Testing of an Innovative Short-Term Large Wind Ramp Forecasting System[C]. European Wind Energy Conference and Exhibition, Warsaw, Poland, 2010.

[7] ArthurBossavy, Robin Girard, George Kariniotakis. Forecasting ramps of wind power production with numerical weather prediction ensembles[J]. Wind Energy, 2013, 16(1):51-63.

[8] Beatrice Greaves, Jonathan Collins, Jeremy Parkes, et al. Temporal Forecast Uncertainty for Ramp Events[J]. Wind Engineering, 2009, 33(4):309-319.

[9] Jiang Wen, Yan Zheng, Yang Jianlin. Reliability assessment of composite generation and transmission system considering wind farms[J]. Power System Protection and Control, 2010 (22): 126-130.

[10]ZHANG Shuo, LI Gengyin, ZHOU Ming. Reliability Assessment of Generation and Transmission Systems Integrated With Wind Farms[J]. Proceedings of the CSEE, 2010, 30(7): 8-14.

[11]CHEN Shuyong, DAI Huizhu, BAI Xiaomin, et al. Reliability Model of Wind Power Plants and Its Application[J]. Proceedings of the CSEE, 2000,(03): 27-30.

[12]Ma H, Liu Y. Real-time recognition of wind power ramp events[C]//Renewable Power Generation Conference (RPG 2013), 2nd IET. IET, 2013: 1-4.

[13] Xu C, Wei Z, He C, et al. Risk Assessment of Wind Power Ramp Event Considering Time-sequence Characteristic[J]. Power and Energy Engineering Conference(APPEEC), 2014: $1-6$.

[14]Wang Chengliang, Zhao Yuan, Zhou Jiaqi, et al. Probabilistic evaluation of power system operation risk based on daily generation scheduling. Automation of Electric Power Systems, 2008, 32(4):6-10.

[15]SUN Rongfu, ZHANG Tao, LIANG Ji. Evaluation and Application of Wind Power Inegration Capacity in Power Grid[J]. Automation of Electric Power Systems, 2011, 35(4): 70-76.

[16]ZHANG Ning, ZHOU Tian-rui, DUAN Chang-gang, et al. Impact of Large-Scale Wind Farm Connecting With Power Grid on Peak Load Regulation Demand[J]. Power System Technology, 2010,(01): 152-158. 